\title{
DE ONDINAS, MELUSINAS, SIRENAS Y PECES. UNA REFLEXIÓN SOBRE MUJER, LENGUAJE Y PODER EN ALGUNAS OBRAS DE LA LITERATURA ALEMANA DEL SIGLO XX
}

\author{
Miriam Palma Ceballos \\ Universidad de Sevilla
}

\begin{abstract}
El agua ha sido, en muchas culturas, un elemento frecuentemente asociado con la feminidad que ha dado lugar a una profusa cristalización en símbolos. Las sirenas, Melusina y las ondinas han sido, en este sentido, figuras relevantes, conectadas, por lo general, con una idea amenazante de lo femenino, si bien en el romanticismo y el surrealismo se revierte en parte dicha equivalencia: aunque igualmente vinculada con lo líquido, se dota a la mujer de un poder redentor. Desde posicionamientos feministas occidentales, sobre todo de la diferencia, la relación entre lo femenino y lo fluido ha servido también para replantear, de modo crítico, el lugar que ocupa la feminidad en el orden simbólico para, desde ahí, esbozar otras posibilidades de conceptualización y representación. El presente artículo se centra en el análisis de algunas de estas figuras acuáticas femeninas en cuatro obras de escritoras del siglo XX pertenecientes al ámbito literario germanoparlante: Ingeborg Bachmann, Irmtraud Morgner y Yoko Tawada. En todas ellas son centrales las figuras asociadas al medio acuático y sirven para abordar cuestiones que tienen que ver con la identidad femenina, el poder, el lenguaje y la escritura.
\end{abstract}

PALABRAS CLAVE: feminismo, mujer y escritura, literatura alemana del siglo XX, figuras acuáticas femeninas, el agua.

Of Melusines, Sirens, Ondines and Fish. A Reflection on Women, Language and Power in Selected Works from Twentieth-Century German Literature

Water has often been linked to femininity in various cultures. This has resulted in a profusion of symbols. Sirens, the figure of Melusine, as well as ondines, have all been relevant figures, associated, for the most part, with a threatening view of the feminine. However, romanticism and then surrealism would subvert such an idea: by linking women to that which is liquid, feminine figures were endowed with redeeming powers. Western feminism, especially that which postulates difference, has employed such an involvement between the feminine and the fluid to critically re-think the role of femininity within the symbolic universe and, stemming from there, to put forth other possibilities of conceptualization and representation. This paper explores some of the aquatic figures present in four works by German-speaking women writers: Ingeborg Bachmann, Irmtraud Morgner, and Yoko Tawada. Figures associated with the aquatic medium are central to all of them, and are deployed to address issues of identity, power, language, and writing.

KEY WORDS: feminism, women and writing, twentieth-century German literature, feminine aquatic figures, water.

Palma Ceballos, Miriam (2021), "De ondinas, melusinas, sirenas y peces. Una reflexión sobre mujer, lenguaje y poder en algunas obras de la literatura alemana del siglo XX", Lectora, 27: 275-289. ISSN: 1136-5781 D.O.I.: 10.1344/Lectora2021.27.14, palma@us.es Recepció: 15 de desembre de 2020 - Acceptació: 21 d'abril de 2021 
¿Acaso el verde de la tristeza y la herrumbe no es la canción de las sirenas? -ANDRÉ BRETON, Los campos magnéticos (1920)

\section{El elemento agua y los personajes acuáticos femeninos en la cultura y la tradición literaria. Introducción y objetivos}

$\mathrm{Al}$ principio de los tiempos, según el himno 129 de la creación del Rig Veda, en el espacio primigenio "todo era agua indiferenciada" (Tola y Dragonetti, 2008: 97). Sobre ese espacio desciende el deseo. Y con él emerge la semilla de la diferenciación. También en el Génesis el agua es el elemento que domina antes de toda gestación. Sobre ella aletea "un viento de Dios" y es a partir de su división en aguas sobre el firmamento y debajo de él, cuando de estas últimas surgen la "tierra" y el "mar" (Biblia de Jerusalén, 2018: 13). Así pues, el agua es, en esas y en otras numerosas tradiciones culturales, elemento asociado a lo originario, a aquello previo a la realidad de las diferencias. Es el ámbito infinito de todo lo posible, "fuente de vida, medio de purificación y centro de regeneración" (Chevalier y Gheerbrant, 2007: 52-64); pero también aparece asociada a menudo al caos, al mal, a la muerte y a las fuerzas fecundas o monstruosas que subyacen lo consciente (56-60). Asimismo, de nuevo en culturas diversas, el agua se concibe como un elemento femenino. Por ese motivo, la mujer, a menudo identificada con la naturaleza, ha sido también frecuentemente asociada a dicho elemento (Puleo, 1992: 78-79). En la tradición occidental, ya en la época griega, una de las diosas principales, la diosa Afrodita, diosa de la irreprimible fuerza del deseo sexual, del placer erótico (Chevalier y Gheerbrant, 2007: 51-52), surge del semen derramado por Urano sobre el mar. Por lo demás, el mundo de las aguas simbólicas de la Grecia clásica está frecuentemente poblado por seres femeninos, siendo las ninfas - oceánidas, nereidas y náyades- las más relacionadas con el medio acuático. Desde entonces, los seres acuáticos femeninos han seguido produciendo una fascinación que no se ha atemperado y se han ido cristalizando en diversos personajes de leyendas, mitos, sagas y cuentos. Las figuras de la tradición grecolatina han pervivido a lo largo de los siglos, y a ellas se han ido sumando otros seres legendarios femeninos surgidos de otras mitologías y tradiciones populares en numerosos puntos de la geografía europea, a los que con frecuencia se les atribuye también la capacidad de atraer a los hombres y llevarlos a la muerte.

La asociación simbólica entre lo femenino y lo fluido es también parte consustancial de las teorizaciones sobre la écriture féminine que realizaron figuras tan relevantes del feminismo francés de la diferencia a partir de los años ochenta como Hélène Cixous o Luce Irigaray. Dicha supuesta escritura es considerada por estas autoras como capaz de subvertir el orden simbólico masculino, un orden cultural en el que se ha ido articulando la diferencia de un modo en el que lo femenino es 
irrepresentable. Para las mujeres - otredad, espejos del hombre, continentes oscuros o relegadas al margen-, en ese orden simbólico es imposible encontrar su verdadera voz. Se hace necesario, por tanto, desde su punto de vista, desarticularlo y propiciar la emergencia de otro en el que lo femenino pueda simbolizarse. Y justamente ese será, según las teóricas de la diferencia, el potencial de la escritura femenina: "Un texto femenino no puede ser más que subversivo: si se escribe, es trastornando, volcánica, la antigua costra inmobiliaria" (Cixous, 1995: 61). Más allá de las diferencias y particularidades de sus respectivos posicionamientos teóricos y discursivos, las teóricas coinciden en atribuir a esa escritura características que tienen que ver con lo líquido. La mecánica de los fluidos servirá a Irigaray para dar cuenta de lo que ella considera como parler femme: lo que dice una mujer es "continuo, comprimible, dilatable, viscoso, conductible, difundible..." (Irigaray, 2009: 83). ${ }^{1}$ Y de modo similar expresa Cixous los atributos de la voz que emanan de la diferente economía femenina reflejada en una escritura fluida, múltiple, ajena a las clasificaciones y a la individuación. Esa voz posibilita la emergencia de lo reprimido y relegado al subconsciente y es capaz de subvertir, transgredir y desbaratar las leyes del orden simbólico propio de la economía masculina (1995: 54-66).

Por su parte, con el resurgimiento de la conciencia feminista en la mitad del siglo $\mathrm{XX}$, no son pocas las autoras que se han apropiado del legado de las figuras acuáticas femeninas. Ofrecen con ello relecturas de esa tradición para abordar cuestiones que tienen que ver con la identidad femenina, la posición simbólica de lo femenino y la posición social de las mujeres. El estudio de la representación de dichas figuras en el ámbito de la literatura alemana ha sido objeto de numerosas investigaciones. Relevantes como punto de partida para el presente análisis son las de Inge Stephan (1987), Barbara Stamer (1987), Claudia Steinkämper (2007) y Andreas Kraß (2010). En ninguna de ellas se aborda la literatura del siglo XX. Es Axel Goodbody (2008) quien ofrece por vez primer un análisis del significado de la aparición de esas figuras en la obra de escritoras alemanas del siglo Xx. Por mi parte, ya realicé con anterioridad una serie de trabajos centrados en el análisis de obras de las autoras que se tratan aquí (Palma Ceballos, 2002, 2008, 2009, 2010, 2011). A partir de las citadas investigaciones, la presente aportación, en la que se lleva a cabo una primera aproximación al tema, intentará analizar qué tiene de semejante y de específico en cada una de las obras tratadas la apropiación de esas figuras asociadas al medio acuático y de qué modo sirve, en cada uno de los casos, para abordar cuestiones que tienen que ver con la identidad femenina, el poder, el lenguaje y la escritura.

\footnotetext{
1 Toril Moi tacha de "infame" dicho fragmento y se pregunta si dicho posicionamiento no es en el fondo contraproducente (1988: 153).
} 


\section{“Undine" de Ingeborg Bachmann}

La primera de las autoras de la que nos ocupamos, la austriaca Ingeborg Bachman (1926-1973), se centra en la figura de la ondina en un texto de 1961: "Undine". Se trata de uno de los relatos que componen la colección que lleva por título Das dreißigste Jahr. Con una clara relación intertextual con la obra del romántico Friedrich de la Motte Fouqué, Undine. Eine Erzählung (1811), el texto de Bachman es un corto monólogo de la ninfa, mezcla de imprecación, lamento, y hasta cierto punto alabanza, en el que Undine se despide del amado, y con ello del mundo humano, y vuelve al medio acuático después de aceptar el fracaso del amor. La figura en la que Fouqué $(2012)^{2}$ se inspira había sido perfilada por Paracelso en su tratado Liber de nymphis, sylphis, pygmaeis et salamandris, et de caeteris spiritibus como el espíritu elemental habitante del agua. Un ser que, si bien similar al humano, no posee alma inmortal, porque, según Stephan, los seres elementales no están enajenados de la naturaleza, de cuyos secretos pueden hacer partícipes a los hombres con los que entran en contacto (1987: 137-139). Con ellos establecen relaciones erótico-amorosas porque solo de ese modo pueden obtener un alma. La unión no podrá disolverse si no media un consenso, por lo que el hombre que tome esa decisión unilateralmente morirá. El ser humano tampoco puede ofender a la ninfa cerca de su medio, so pena de ser castigado. Justo este es el destino final de Huldebrant al incumplir ambas reglas: rompe la unión y se olvida de Undine, pese a haberla desposado, para casarse con su anterior prometida, Bertalda. De este modo, se muestra cómo la humanización de la ninfa se logra solo a costa de su domesticación, de su adaptación al estereotipo social burgués femenino de la época: mansa, moral y pendiente del hombre (Steinkämper, 2007: 231). Dorowin interpreta esta obra de Fouqué como un intento de configurar la utopía romántica de la unión de contrarios, entre naturaleza y cultura, hombre y mujer, razón y sentimiento; una utopía que, pese a todo, acaba fracasando, porque la otredad acaba siendo considerada como una amenaza para la estabilidad de la estructura social (2000: 2). Según considera Stephan, en dicha obra Fouqué intenta tematizar la imposibilidad de aunar erotismo y compromiso en el matrimonio burgués, como sucede en otros autores románticos, impedimento que, según analiza, surge de la voluntad de poder de un sujeto masculino que convierte a la mujer imaginada como ser natural en objeto de dominio. La identificación de la mujer con naturaleza contiene así, pese a su intención utópica, una sutil dosis de violencia (1987: 134).

Gran parte de estas ideas aletean en la obra de Ingeborg Bachmann. Un ansia de unión a través del amor total es también lo que vertebra el lamento de despedida

\footnotetext{
${ }^{2}$ La obra de Fouqué es la más famosa de la época, pero no la única. Los amores trágicos entre ninfas y caballeros estaban de moda en la época del Romanticismo (Kraß, 2010: 239).
}

278

Lectora, 27 (2021): 275-289. ISSN: 1136-5781 D.O.I.: 10.1344/Lectora2021.27.14 
de la ondina de la autora austriaca. Pero en este relato es la ninfa quien toma la palabra y realiza una certera radiografía del reparto de posiciones de lo femenino y lo masculino en un sistema que impide, finalmente, alcanzar esa unión ansiada. El texto puede leerse, por ello, como la expresión del duelo de un yo femenino que ha comprendido, y finalmente aceptado, la inevitabilidad de esa realidad. El discurso, abiertamente contradictorio, ambiguo y, a primera vista, muchas veces alógico, comienza con la imprecación a un género humano que siempre lleva, dice, el nombre de Hans: "Ihr Menschen! Ihr Ungeheuer!". ${ }^{3}$ Estos reiterados apelativos se dirigen a un género masculino al que se perfila como responsable $y$, al mismo tiempo, víctima de su incapacidad de amar, de acercarse al mundo y a la otredad de otro modo que no sea a través del cálculo de la rentabilidad y el interés egoísta. Pero este hombre es, a la vez, casi al final del discurso, también alabado por ser capaz de actos de heroísmo, ternura y sacrificio y de generar un conocimiento y un progreso que provocan admiración. En ocasiones, además, se narra cómo en él habita la nostalgia, reprimida pero latente, de volver al elemento agua y a los momentos, especiales y escasos, en los que, en contacto con la conciencia de su propia finitud, de su vulnerabilidad y de su soledad, se acerca a sí mismo. Son esas las oportunidades en las que puede producirse un verdadero encuentro. Frente a la figura de Fouqué, la ondina de Bachmann no va a ser domesticada, no pierde la conciencia de su autonomía ni, pese a todo, siente que su tarea sea la de existir para el hombre que ama: "Ich bin nicht gemacht, um eure Sorgen zu teilen. Diese Sorgen nicht!" (Bachmann, 1966: 144). ${ }^{4}$ Y, en cierto modo, se describe como provista de un grado de conciencia y comprensión más elevado que el del hombre, lo que le permite conocerlo y comprenderlo más allá de las apariencias. Con todo, ella será siempre la otra, la que conserva el lado salvaje, pero con la que los hombres no permanecen porque establecen vínculos formales con las mujeres a las que utilizan. Interesante es en este texto también la importancia que adquiere el lenguaje. El elemento de la ninfa, el agua, es un no-lugar en el que no existe el lenguaje, que surge solo en contacto con lo humano, pero está impregnado asimismo de la misma reificación que el resto de la producción humana. Sin embargo, aletea en el discurso de Bachmann la posibilidad de otro lenguaje diferente, verdadero, que surge en esos momentos de epifanía en los que la consciencia de la finitud impide al hombre agarrarse momentáneamente a otras tablas de salvación. Y esa verdad es la que intenta materializarse en el mismo discurso textual, en una prosa que rezuma un profundo lirismo y en la que a través de esa intercalación rítmica de

\footnotetext{
3 “¡Vosotros, hombres! ¡Vosotros, monstruos!” (Todas las traducciones de las citas de las obras que se incluyen en este artículo son propias).

4 "No estoy hecha para compartir vuestras preocupaciones. ¡No esas!”.
} 
elementos ambivalentes — nostalgia y hastío, amor y repulsión, comprensión y rabia- se simula la fluidez del movimiento de las olas (Dorowin, 2000: 4).

\section{Melusina y las sirenas en Irmtraud Morgner}

Por lo que respecta a la segunda de las autoras, toda la producción literaria de la escritora de la RDA Irmtraud Morgner (1933-1990), salvo sus dos primeras obras, se concentra en temas relacionados con la cuestión femenina. Perfilar un sujeto de posibles proyectos emancipatorios y convertir a la mujer en hacedora de historia se erigen, por ello, en objetivos fundamentales de su búsqueda literaria. Fruto de ese empeño es el ambicioso proyecto de una trilogía truncado por su temprana muerte. En la primera de las novelas que la componen, Leben und Abenteuer der Trobadora Beatriz nach Zeugnissen ihrer Spielfrau Laura (1974), se narra la compleja relación entre Laura Salman, una ciudadana de la RDA, y la trovadora Beatriz, una recreación de Beatriz de Dia (1140-1175), representante de un pequeño grupo de mujeres poetas, las trobairitz, quienes, invirtiendo los códigos del amor cortés, compusieron sus propios poemas en los que un yo femenino se erigía en sujeto del deseo amoroso y en los que el hombre se convertía en el objeto deseado.

En la ficción de Morgner, Beatriz es bruscamente despertada en el agitado periodo de las revueltas estudiantiles del ' 68 , tras un largo sueño voluntario de ochocientos años. Toma dicha decisión esperando poder despertar en una época en la que el hecho de ser mujer no supusiera un impedimento para el ejercicio literario. La estructura de la obra, una "novela operativa de montaje", es extremadamente compleja por lo que respecta, entre otras cosas, a la mezcla de elementos reales y fantásticos, al continuo juego de voces y de perspectivas y a la falta de linealidad narrativa debido a la introducción de elementos que rompen la unidad (Palma Ceballos, 2011: 100). Una figura relevante de este artefacto literario es la figura de Melusine, personaje rescatado, como Beatriz, de la tradición literaria, pero legendario en este caso. Con su introducción en el juego de la ficción se permite reescribir el significado de una figura femenina acuática de origen indeterminado, aparecida en la Edad Media e intensamente recreada entre los siglos XII y XVI. En casi todos los textos, el hada se casa con el caballero a condición de que este se comprometa a no verla en un determinado día, generalmente el sábado. Ese día Melusina se recluye en un su recinto privado y se transforma en un ser con torso de mujer y cola de dragón o serpiente. Una obra que sin duda tiene en cuenta Morgner es Melusine, una novela escrita en 1496 por Thüring von Ringoltingen (Kraß, 2010: 79-96). Paracelso también considera a Melusina como ninfa, pero le atribuye, frente a la ondina, una dualidad que incluye el aspecto oscuro, negativo de la feminidad; presenta, según él, atributos y comportamientos reprobables que bien permiten caracterizarla como bruja (Steinkämper, 2007: 209). Y es así, como una demoníaca seductora, como será recibida y reproducida en muchas de las 
obras en la época moderna, siendo la recepción de la figura especialmente fructífera en el Romanticismo, aunque probablemente no de modo directo, sino con medición de otros autores anteriores, Jakob Böhme entre ellos. En el Romanticismo, Melusina será la protagonista de obras de autores como Ludwig Tieck y su Sehr wunderbare Historie von der Melusina (1800), donde adquiere rasgos de sirena. La figura también suscita el interés de Goethe. En su novela corta Neue Melusine (aparecida antes, pero insertada finalmente en Wilhelm Meister Wandersjahre en 1821) rompe totalmente con la tradición y la convierte en una enana. De este modo se transforma en un espíritu de la tierra. Su Melusina es, además, una talentosa cantante e intérprete de laúd. También aquí se remite al mito de las sirenas de Ulises (Kraß, 2010: 100-125). En el siglo XIX se lleva a cabo una transformación y adquiere rasgos de mujer autoconsciente y libre (125-131).

En esta última línea se sitúa el personaje de la novela de Irmtraud Morgner, en cuyo universo ficticio la figura se transforma y se resignifica totalmente. Alejándose de la carga simbólica tradicional, su Melusine ya no corporeizará una feminidad imaginada desde el punto de vista masculino ni será sujeto de utopías regresivas. Conserva los rasgos físicos propios de la figura legendaria, sigue siendo una mezcla de mujer y dragón, pero en la novela se incide, sin embargo, en su capacidad de volar: Melusine abandona el medio acuático y es asociada simbólicamente a un elemento, el aire, que representa la actividad y la independencia (Detken, 2005: 135). Ya no será la seductora que acarrea la desgracia al marido en el caso de que este rompa la promesa inicial. Desmitificada de ese modo, autónoma y libre, la Melusine de Morgner es una activista política, miembro, junto con su cuñada Beatriz, de una facción feminista de su paródica invención de la historia de la lucha por la emancipación femenina. Este grupo, en oposición al liderado por Perséfone y Deméter, que promulgan una vuelta del matriarcado, se decanta por el tercer orden: "Die weder patriarchalisch noch matriarchalisch sein sollte, sondern menschlich" (Morgner, 1976: 20). ${ }^{5}$ De este modo, corporeiza, hasta cierto punto, el posicionamiento ideológico de la propia autora en el momento de su composición (Palma Ceballos, 2011: 92). Pero, además de ello, es una voz importante en la novela. Como cronista y responsable de parte de los textos, permite abrir espacios de reflexión sobre la relación de las mujeres, la escritura y la memoria: la figura de Melusine se presenta como la autora del Melusinisches Buch, un heterogéneo compendio de textos procedentes de otras mujeres que se insertan como intermezzos entre los otros capítulos de este experimento literario de la autora socialista. Entre ellos figuran los extractos, ligeramente modificados y adaptados al nuevo marco, de la novela anterior de la propia Morgner, Rumba aufeinen Herbst, censurada en su momento y que no pudo aparecer tras su composición, en

\footnotetext{
5 “Que no debía de ser ni patriarcal ni matriarcal, sino humano".
} 
1966. Con este nuevo juego de autorías en la ficción se intenta salvaguardar ella misma de las consecuencias de la misma lógica del olvido que subyace la escritura de la historia y el establecimiento del canon literario que ha acallado o silenciado la experiencia, la voz y la escritura femeninas. Es esta una labor que constituye el motor fundamental de la novela (Detken, 2005: 142).

La segunda de las novelas de la trilogía, Amanda, ein Hexenroman (1983), en la que Laura y Beatriz vuelven a ser las protagonistas, es un proyecto aún más ambicioso, si cabe. En ella Beatriz resucita de nuevo tras la muerte accidental en la anterior novela. Esta vez lo hace como sirena que ha perdido la voz. La tarea que le será encomendada será la de "cantar", es decir, reescribir, confinada en una jaula del zoológico berlinés, la verdadera historia de Laura Salman.

En esta novela, de nuevo compleja, caleidoscópica, las sirenas forman parte de un mundo mítico. Es este uno de los niveles narrativos que se articulan en su polifónica estructura. Con esta figura, Morgner conecta su ficción con el origen de nuestra tradición literaria. Como las sirenas homéricas, las de Amanda, ein Hexenroman no tienen la forma que adquirieron en la tradición medieval. Porque las primigenias, aunque asociadas al ámbito marítimo, no tenían forma de pez, sino de pájaro. Con esta forma no se distinguirán sino bastante después de que las sirenas de La Odisea regresen al agua, tras su intento fracasado de seducir al héroe con su canto, cuando renazcan en la tradición cristiana ${ }^{6}$ y comience su transformación. Las sirenas de la iconografía medieval ya comienzan a aparecer como mitad peces mitad mujeres. Con el cambio icónico, la figura empieza a tener una carga sexual muy acusada (Rodríguez Peinado, 2009: 56-57). Y en los textos, las sirenas aparecen una y otra vez a lo largo de toda la historia literaria europea. En ellos también conviven durante un tiempo sirenas-pájaro, sirenas-pez y sirenas con mezcla de ambos hasta que definitivamente se impone la figura pisciforme. De cualquier modo, en todos los casos la voz es su atributo consustancial (Kraß, 2010: 47-76).

Morgner lleva a cabo de nuevo una profunda resignificación del mito. En la propia cosmogonía que se ofrece en la ficción de la obra, las sirenas son las hijas de la madre Tierra, encerrada por el padre, Aire, para asegurarse la legitimidad de la descendencia. En el principio de la deriva violenta y patriarcal de la civilización está, por tanto, la dualidad, gestada y sostenida por la entronización de la razón instrumental en el sistema de pensamiento occidental. Razón y naturaleza se hacen irreconciliables. La subordinación de las mujeres, piensa Morgner en consonancia visible con la crítica a la racionalidad de los teóricos de la Escuela de Frankfurt (Adorno y Horkheimer, 1984), es una realidad íntimamente conectada con esa dicotomía: razón tecnológica y patriarcal son identificables (Palma Ceballos, 2011:

\footnotetext{
${ }^{6}$ Según Andreas Kraß, el primer testimonio que se puede rastrear es la descripción de una mujer pez en un bestiario del siglo VII, el Liber Monstrorum (2010: 61).
}

282

Lectora, 27 (2021): 275-289. ISSN: 1136-5781 D.O.I.: 10.1344/Lectora2021.27.14 
182). Conforme a ello, frente a la tradición, y también de acuerdo con la interpretación que Adorno y Horkheimer le dan al periplo de Ulises, el poder de su voz no es destructivo: las sirenas han ido enmudeciendo a medida que el orden racional y patriarcal se ha ido consolidando:

In alten Zeiten lebten alle weisen Frauen ein zweites Leben in Sirenengestalt. Damals gab es viele Sirenen. Dann übernahmen die Männer die Herrschaft und führten ein, was die Menschen heute Geschichte nennen: Privateigentum, Klassentrennung, Ausbeutung, Staatsgewalt, Kriege. Kriegslieder konnten die Sirenen mühelos niedersingen. In Kriegen verstummten die Wesen. Danach erinnerten sie sich wieder und gewannen ihre Sprache zurück. Als jedoch die Zeiträume zwischen den Kriegen kürzer und kürzer wurden, blieb den Sirenen keine Zeit mehr zum Erinnern. Auch wurden die weisen Frauen immer seltener. In Küchen können keine wachsen. Und in Kriege können keine auferstehen... (Morgner, 1983: $12-13)^{7}$

Por ello, esa voz relegada históricamente, anterior al lenguaje de la razón instrumental, es la que se considera capaz de contrarrestar la tendencia violenta y autodestructiva que caracteriza a ese estadio de la civilización. Dada la deriva de la civilización (el patriarcado, los conflictos bélicos y el peligro de la destrucción ecológica del planeta), esa necesidad se hace perentoria. Por esa razón, a Beatriz se le impone la tarea de la reescritura de la historia de Laura, al considerarse que la anterior novela "stinkt nach innerer Zensur" (Morgner, 1983: 18) ${ }^{8} \mathrm{y}$, sobre todo, porque, como sentencia Arke, escribir (recordar la voz) es, dada la premura histórica, una obligación: "Wenn plötzlich Sirenen auftauchen, haben sie die Pflicht, zu singen” (Morgner, 1983: 24). ${ }^{9}$ Como se analiza profusamente en La construcción del sujeto femenino en la obra de Irmtraud Morgner, la idea de este poder que la sirena Arke atribuye a la voz de las sirenas entronca también con teorizaciones como las de Herbert Marcuse (1995), los feminismos de la diferencia y los planteamientos

\footnotetext{
7 "En tiempos remotos todas las mujeres sabias vivían una segunda existencia en forma de sirenas. Entonces los hombres asumieron el poder e iniciaron lo que hoy por hoy la humanidad denomina historia: propiedad privada, división de clases, explotación, terror estatal, guerras. Los cantos bélicos acallaban a las sirenas sin demasiado esfuerzo. En las guerras enmudecían. Después comenzaban a recordar y recuperaban su lenguaje. Sin embargo, a medida que los intervalos entre las guerras se hacían más y más cortos, les quedaba cada vez menos tiempo para recordar. Al tiempo las mujeres sabias eran cada vez menos frecuentes. En las cocinas no pueden crecer. Y en las guerras no pueden resucitar".

8 "Apesta a censura interna".

9 "Cuando de repente aparecen sirenas, tienen la obligación de cantar".
} 
ecofeministas. Desde diferentes puntos de vista, todos parten de la idea de que es precisamente la marginalización de las mujeres en la tarea de construcción cultural lo que las puede convertir en el sujeto revolucionario de las transformaciones culturales, sociales, políticas y económicas alternativas y necesarias para evitar las catastróficas consecuencias a las que se ve abocada la sociedad vertebrada por el principio de la razón instrumental y/o el pensamiento androcéntrico (Palma Ceballos, 2011: 61-70,180-192). ${ }^{10}$ Esa voz que busca recordar la sirena Beatriz sería la que corresponde a lo que la autora denomina como lenguaje concreto, contrapuesto al lenguaje abstracto. Con ello parece apuntar a la existencia de un modo de comunicación diferente al dominante, cercano en gran medida a esa voz postulada por las teóricas de la escritura femenina antes citadas, que conservaba algo de lo primigenio, lo presimbólico, anterior a la individuación y que se identifica como femenina: "La voz, canto anterior a la ley, antes de que el aliento fuera cortado por lo simbólico, reapropiado en el lenguaje bajo la autoridad que separa. La más profunda, la más antigua y adorable visitación. En toda mujer canta el primer amor sin nombre" (Cixous, 1995: 57). La voz de las sirenas del universo de Morgner busca, por lo tanto, dar cuenta de un modo de relación con la realidad cercano a la experimentación poético-mítica de la naturaleza. En la obra, este modo de vinculación está basado en la integración y no en la dominación. Y ha sido un modo de expresión relegado y reprimido históricamente a medida que se ha ido entronizando un tipo de pensamiento racional basado en la estricta e insalvable separación entre sujeto y objeto, razón y naturaleza, responsable, también, de la histórica subordinación de lo femenino (Palma Ceballos, 2011: 61-70, 180-192).

\section{El pez de Yoko Tawada}

Igualmente complejo es el entramado discursivo con el que se narran las metamorfosis que sufre la protagonista de la última de las obras analizadas aquí, Das Bad, de la escritora japonesa Yoko Tawada, afincada en Alemania desde 1982. Lo fluido forma parte sustancial de la obra en todos los niveles. Y es justamente el agua el elemento fundamental del comienzo de la novela cuando la protagonista se mira al espejo:

Der menschliche Körper soll zu achtzig Prozent aus Wasser bestehen, es ist daher auch kaum verwunderlich, daß sich jeden morgen ein anderes Gesicht im Spiegel zeigt. Die Haut an Stirn und Wangen verändert sich

\footnotetext{
${ }^{10}$ Goodbody (2008: 244) considera a Morgner como ecofeminista. Pese a que es innegable que alguno de los niveles discursivos de la novela está cercano a esta corriente, lo cierto es que la complejidad de voces y discursos que se entretejen en Amanda, ein Hexenroman imposibilita un estricto encasillamiento del feminismo de Morgner (Palma Ceballos, 2011: 180).
}

284

Lectora, 27 (2021): 275-289. ISSN: 1136-5781 D.O.I.: 10.1344/Lectora2021.27.14 
von Augenblick zu Augenblick wie der Schlamm in einem Sumpf, je nach der Bewegung des Wassers, das unter ihm fließt, und der Bewegung der Menschen, die auf ihm ihre Fußspuren hinterlassen. ${ }^{11}$ (Tawada, 1993) ${ }^{12}$

A partir de ese momento, narra una serie de metamorfosis corporales: en su primera transformación, su piel se va cubriendo de escamas, consigue deshacerse de las mismas con un baño, pero vuelve después a cubrirse definitivamente de ellas. El motivo del pez se reitera a lo largo de todo el relato y se convierte, de ese modo, junto con el del espejo y el de la muerte, en parte sustancial del mismo (Palma Ceballos, 2010: 122-123). Los guiños que hace la voz narradora en sus sucesivas transformaciones a los seres femeninos acuáticos de la tradición europea de los que tratamos en este ensayo son patentes en este texto. Pero también hay referencias a la mujer pez de la tradición japonesa que, según analiza Sabine Fischer, simboliza la mediación entre el amorfo mundo natural y la civilización (1997: 106). Especialmente interesante es señalar las coincidencias con los textos que Breton, bajo el título El pez soluble, publicó en 1924 junto al Manifiesto surrealista. En el discurso que narra el onírico periplo del yo del enunciado, la presencia femenina o su cuerpo, a menudo fragmentado, es constante; así como lo es también el elemento agua (Breton, 1985: 71-145). Como señala Alicia Puleo, en el surrealismo se revierte el valor negativo que había tenido la equiparación de la ecuación feminidad, naturaleza e inconsciente. Para Breton el erotismo adquiere un estatuto gnoseológico. Se lleva a cabo una revalorización de la femineidad, encarnada fundamentalmente en la figura de la femme-enfant. La mujer, lo absolutamente otro, se considera el medio a través del cual el hombre conecta con el inconsciente, vía privilegiada para llegar a lo absoluto (Puleo, 1992: 73-79). El discurso de Das Bad bien podría considerarse emparentado al utilizado por la vanguardia de comienzos del siglo. También en Das Bad se diluyen las fronteras entre lo onírico y lo real en un estilo metafórico y metonímico para dar cuenta de la delirante transformación de la protagonista. Sin embargo, frente a la postura de Breton, la intención del discurso de Tawada es deconstruir a través de esos procedimientos discursivos, de un modo irónico, la visión de la feminidad predominante, incluida aquella que le atribuye una función redentora, como la atrapada en el propio discurso literario del surrealismo. Su compleja simbología, la transformación en pez y las otras metamorfosis que sufre la protagonista extranjera muestran la imposibilidad de acceso a la corporalidad a través de un lenguaje en el que el cuerpo femenino es un cuerpo

\footnotetext{
11 "El cuerpo humano se compone en un ochenta por ciento de agua. Por eso apenas es sorprendente que cada mañana se muestre en el espejo una cara distinta. La piel de la frente y de las mejillas cambia a cada momento, como el fango de los pantanos, según el movimiento del agua que fluye por debajo y el movimiento de las personas que dejan sus huellas sobre él”.

12 El libro no está paginado.
} 
interpretado, codificado como superficie sobre el que el sujeto masculino proyecta su deseo. Ello no impide, sin embargo, que el propio proceso de enajenación tematizado sea en sí mismo también un acto de rebeldía contra la posición de alienada y colonizada. Su escritura fluida, desbordante, metamórfica constituiría así un intento de cuestionar y quebrantar las reglas del orden simbólico y cultural que sustenta la construcción de los significados y de las propias subjetividades y en el que el sujeto normativo solo puede poseer, en el discurso de Tawada, rasgos identitarios masculinos. Podría pensarse, entonces, que la obra de Tawada puede categorizarse como écriture féminine. Sin embargo, en mi opinión, la posición irónica de la escritora japonesa también intenta desenmascarar lo infructuoso de ese empeño. La voz que finalmente pronuncia el discurso es la de una mujer muerta que acompaña a la protagonista después de que ella misma pierda la lengua. Ni siquiera los feminismos occidentales pueden hallar posicionamientos estables y definitivos desde los que se articule una voz femenina. Las mismas teorizaciones feministas corren el peligro de construir sus sujetos femeninos con la misma lógica que intentan deconstruir. Las sucesivas metamorfosis y el flujo del discurso de la protagonista obedecen más bien a una intención paródica y performativa, estrategias utilizadas por su potencial subversivo para poner de manifiesto la falsa naturalidad del binarismo que vertebra el orden en el que se inscriben los géneros y abrir posibilidades para desestabilizarlo.

\section{Conclusiones}

Este breve análisis de las cuatro obras de Ingeborg Bachmann, Irmtraud Morgner y Yoko Tawada ha pretendido mostrar cómo a partir de la segunda mitad del siglo $\mathrm{XX}$, coincidiendo con el resurgimiento de la conciencia feminista, se lleva a cabo por parte de esas escritoras una reapropiación en sus ficciones de los seres acuáticos femeninos que aparecen en la tradición literaria occidental desde sus inicios. En todos los casos, son esas figuras las que toman la palabra en la ficción para, con su propia voz, cuestionar y replantear la relación entre las imágenes tradicionales de la femineidad, su posición en el orden simbólico y el lugar que ocupan las mujeres en la distribución del poder en nuestro sistema social. Dicha crítica y cuestionamiento se materializa asimismo en las características del discurso empleado, en el que la propia voz - el lenguaje - se convierte en objeto de reflexión. La narración presenta atributos de flujo: un discurso fluido, cambiante, alógico en Ingeborg Bachmann; caleidoscópico y polifónico en Irmtraud Morgner; irónicamente metamórfico, desbordado, perfomático y cercano al utilizado en el surrealismo en Yoko Tawada. Pero si a través de ese lenguaje, en las obras de Bachmann y de Morgner parece postularse la existencia de una voz propiamente femenina, Tawada se sirve de dicho registro discursivo precisamente para criticar irónicamente la esencialización de lo femenino. 


\section{REFERENCIAS BIBLIOGRÁFICAS}

Adorno, Theodor W. y Mark Horkheimer (1984), Dialektik der Aufklärung. Philosophische Fragmente. Gesammelte Schriften, tomo 3, Fráncfort del Meno, Suhrkamp.

Bachmann, Ingeborg (1966), "Undine geht”, Das dreißigste Jahr, Múnich, DTV: 140-147.

Biblia de Jerusalén (2018), Bilbao, Desclée de Brouwer.

Breton, André (1985), Manifiestos del surrealismo, Barcelona, Labor.

Chevalier, Jean y Alain Gheerbrant (2007), Diccionario de símbolos, Barcelona, Herder.

Cixous, Hélène (1995), La risa de la medusa, Barcelona, Anthropos.

Detken, Anke (2005), "Weibliche Geschichte und Erinnern. Irmtraud Morgners Leben und Abenteuer der Trobadora Beatriz nach Zeugnissen ihrer Spielfrau Laura", Geschichte(n)-erzählen: Konstruktionen von Vergangenheit in literarischen Werken deutschsprachiger Autorinnen seit dem 18. Jahrhundert, Marianne Henn, Irmela von der Lühe y Anita Runge (eds.), Göttingen, Wallstein Verlag: 125-144.

Dorowin, Hermann (2000), “Ingeborg Bachmann: Undine geht”, Fráncfort del Meno, Universitätsbibliothek Johann Christian Senckenberg. $\quad<$ http://publik ationen.ub.uni-frankfurt.de/frontdoor/index/index/docId/14078>

Fischer, Sabine (1997), “'Verschwinden ist schön'. Zu Yoko Tawadas Kurzroman Das Bad", Denn du tanzt auf einem Seil: Positionen deutschsprachiger MigrantInnenliteratur, Sabine Fischer y Moray McGowan (eds.), Stuttgart, Stauffenburg Verlag: 101-113.

Fouqué, Friedrich de la Motte (2012), Undine. Eine Erzählung, Paderborn, Salzwasser Verlag. [1811]

Goodbody, Axel (2008), "Wasserfrauen in ökofeministischer Perspektive bei Ingeborg Bachmann und Karen Duve: Mahnende Stimmen über unsere Beziehung zur Natur”, Wasser - Kultur - Ökologie. Beiträge zum Wandel im Umgang mit dem Wasser und $z u$ seiner literarischen Imagination, Axel Goodbody y Berbeli Wanning (eds.), Göttingen, V\&R Unipress: 241-264.

Irigaray, Luce (2009), Ese sexo que no es uno, Raúl Sánchez Cedillo (trad.), Madrid, Akal. [1982]

Kraß, Andreas (2010), Meerjungfrauen. Geschichten einer unmöglichen Liebe, Fráncfort del Meno, Fischer.

Marcuse, Herbert (1995), Eros y civilización, Barcelona, Ariel.

Moi, Toril (1988), Teoría literaria feminista, Madrid, Cátedra. 
Morgner, Irmtraud (1976), Leben und Abenteuer der Trobadora Beatriz nach Zeugnissen ihrer Spielfrau Laura. Roman in dreizehn Büchern und sieben Intermezzos, Darmstadt, Luchterhand.

-(1983), Amanda, ein Hexenroman, Darmstadt, Luchterhand.

Palma Ceballos, Miriam (2002), "Lenguaje de sirenas. Mujer, lenguaje y transgresión en Amanda, ein Hexenroman”, Mujer, cultura y comunicación: realidades e imaginarios, Manuel Ángel Vázquez Medel y Mercedes Arriaga (eds.), Sevilla, Universidad de Sevilla.

-(2008), "Porque la palabra hablada se hace carne", Encarna(c)ciones. Teoría(s) de los cuerpos, Meri Torras y Noemí Acedo (eds.), Barcelona, Editorial UOC: 153-160.

-(2009), "Un cuerpo es una palabra es un cuerpo es una palabra... Das Bad de Yoko Tawada: el discurso y los cuerpos de las otras", Matrias, patrias. Identidades traspasando fronteras, Miriam Palma Ceballos (ed.), Madrid, Entimema: 125-143.

-(2010), "Frauen hinter einem Sprachgitter? Frauen, Sprache und Macht im literarischen Diskurs Das Bad von Yoko Tawada”, Die Gläserne Decke: Fakt oder Fiktion. Eine literarische Spurensuche im deutschsprachigen Werken von Autorinnen, Dolors Sabaté y Marion von Schulz (eds.), Fráncfort del Meno, Peter Lang: 241-264.

-(2011), La construcción del sujeto femenino en la obra de Irmtraud Morgner, Sevilla, Universidad de Sevilla.

Puleo, Alicia H. (1992), Dialéctica de la sexualidad. Género y sexo en la filosofía contemporánea, Madrid, Cátedra.

Rodríguez Peinado, Laura (2009), “Sirenas”, Revista digital de iconografía medieval, 1 (1): 51-63.

Stamer, Barbara (ed.) (1987), Märchen von Nixen und Wasserfrauen, Fráncfort del Meno, Fischer Taschenbuch.

Steinkämper, Claudia (2007), Melusine - vom Schlangenweib zur "Beauté mit dem Fischschwanz". Geschichte einer literarischen Aneignung, Gotinga, Vandenhoeck \& Ruprecht.

Stephan, Inge (1987), "Weiblichkeit, Wasser und Tod: Undinen, Melusinen und Wasserfrauen bei Eichendorff und Fouqué", Weiblichkeit und Tod in der Literatur, Inge Stephan (ed.), Colonia, Böhlau: 117-130.

Tawada, Yoko (1993), Das Bad, Tubinga, Verlag Claudia Gehrke.

-(2008), "El Baño”, Miriam Palma Ceballos (trad.), Lectora: revista de dones $i$ textualitat, 14: 333-383.

288

Lectora, 27 (2021): 275-289. ISSN: 1136-5781 D.O.I.: 10.1344/Lectora2021.27.14 
Tola, Fernando y Carmen Dragonetti (2008), Filosofía de la India: del Veda al Vedanta: el sistema Samkhya: el mito de la oposición entre "pensamiento" indio y "filosofía" occidental, Barcelona, Editorial Kairós.

\section{@(ㅇ) $\Theta \Theta$}

